\title{
Infrecuente asociación de coronaria circunfleja anómala y coartación aórtica
}

\author{
Uncommon association of anomalous circumflex coronary and aortic coarctation
}

\author{
Susan C. Pumacayo-Cárdenas* y Regina de la Mora-Cervantes \\ Departamento de Tomografía Computarizada Cardiovascular, Instituto Nacional de Cardiología Ignacio Chávez. Ciudad de México, México
}

Paciente de 25 años de edad, con antecedente de coartectomía y ampliación de arco aórtico a los 2 años de edad. Aortoplastia con stent a los 13 años de edad por recoartación; se encuentra asintomático en reposo y en esfuerzo niega episodios de dolor torácico, disnea y síncope. El examen clínico, ECG y la ecocardiografía fueron normales. La angiotomografía de control para la coartación de aorta, que se obtuvo mediante protocolo de adquisición no sincronizado, demostró stent íntegro, permeable, sin evidencia de recoartación. Como hallazgo incidental se detectó (Fig. 1) origen anómalo de la coronaria circunfleja (CX) de la arteria pulmonar izquier$\mathrm{da}$, con trayecto caudal entre ambas arterias pulmonares, donde da origen a ramo del nodo sinusal y luego recorre medial a la aurícula izquierda para dirigirse hacia el surco atrioventricular izquierdo. Es importante notar que el flujo de la $\mathrm{CX}$ es retrógrado, dado por hiperdensidad que se observa desde la CX hacia la arteria pulmonar. En la reconstrucción volumétrica (Fig. 2A) se observó el stent debajo de la arteria subclavia izquierda y el trayecto de la CX. Es importante notar la circulación coronaria colateral, de la descendente anterior y la coronaria derecha, a través de sus ramos diagonales y agudo marginal, respectivamente (Fig. 2 B y C).

El origen de la $C X$ desde la arteria pulmonar (ACCAPA) es una anomalía coronaria congénita de baja prevalencia $(<0.3 \%)^{1-2}$, y menos frecuente cuando se origina de la arteria pulmonar izquierda ${ }^{2}$. La presentación clínica es muy variable, al ser una patología muy infrecuente dependerá de la circulación coronaria colateral; se ha reportado desde muerte súbita e infarto de miocardio hasta asintomáticos, siendo un hallazgo. La asociación con otras cardiopatías congénitas se presenta en el 3-36\% de los casos, entre las más frecuentes se han descrito aquellas que comprometen la formación embriológica del tronco-cono, como la ventana aortopulmonar, tetralogía de Fallot, conducto arterioso, estenosis pulmonar y coartación de aorta, y de forma más infrecuente el síndrome de cimitarra ${ }^{2-3}$.

Este caso es importante por presentar una patología (ACCAPA) infrecuente y más aún al originarse en la rama pulmonar izquierda, asociada a coartación aórtica; en el contexto de un paciente asintomático, cuyo diagnóstico se realizó mediante una tomografía de seguimiento, no dirigido para coronarias, evidenciándose también la circulación colateral de la coronaria derecha y descendente anterior, que permitió la supervivencia del paciente.

\section{Financiamiento}

La presente investigación no ha recibido ayudas específicas provenientes de agencias del sector público, sector comercial o entidades sin ánimo de lucro.

\section{Correspondencia:}

*Susan C. Pumacayo-Cárdenas

Col. Belisario Domínguez, Sec. XVI, Del. Tlalpan

C.P. 14080, Ciudad de México, México

Fecha de recepción: 10-09-2018

Fecha de aceptación: 01-03-2019

DOI: 10.24875/ACM.M19000023
Disponible en internet: 08-05-2019 Arch Cardiol Mex. 2019;89(2):183-184 www.archivoscardiologia.com

1405-9940 @ 2019 Instituto Nacional de Cardiología Ignacio Chávez. Publicado por Permanyer México SA de CV. Este es un artículo Open Access bajo la licencia CC BY-NC-ND (http://creativecommons.org/licenses/by-nc-nd/4.0/). 

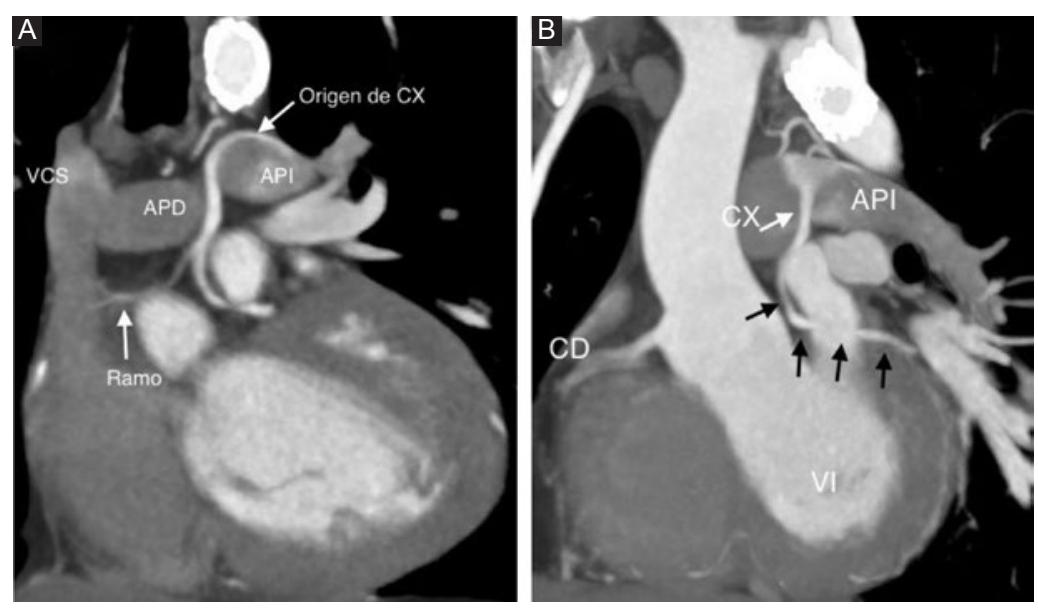

Figura 1. Angiotomografía, protocolo FLASH. A: origen de la coronaria circunfleja (CX) desde parte proximal y cara interna de la API, con trayecto caudal entre API y APD, donde da origen a ramo del nodo sinusal. B: se observa origen y flujo retrógrado de circunfleja hacia API (flecha blanca). Trayecto de CX hacia el surco atrioventricular izquierdo (flechas negras). API: arteria pulmonar izquierda; APD: arteria pulmonar derecha; VCS: vena cava superior; CD: coronaria derecha; VI: ventrículo izquierdo.

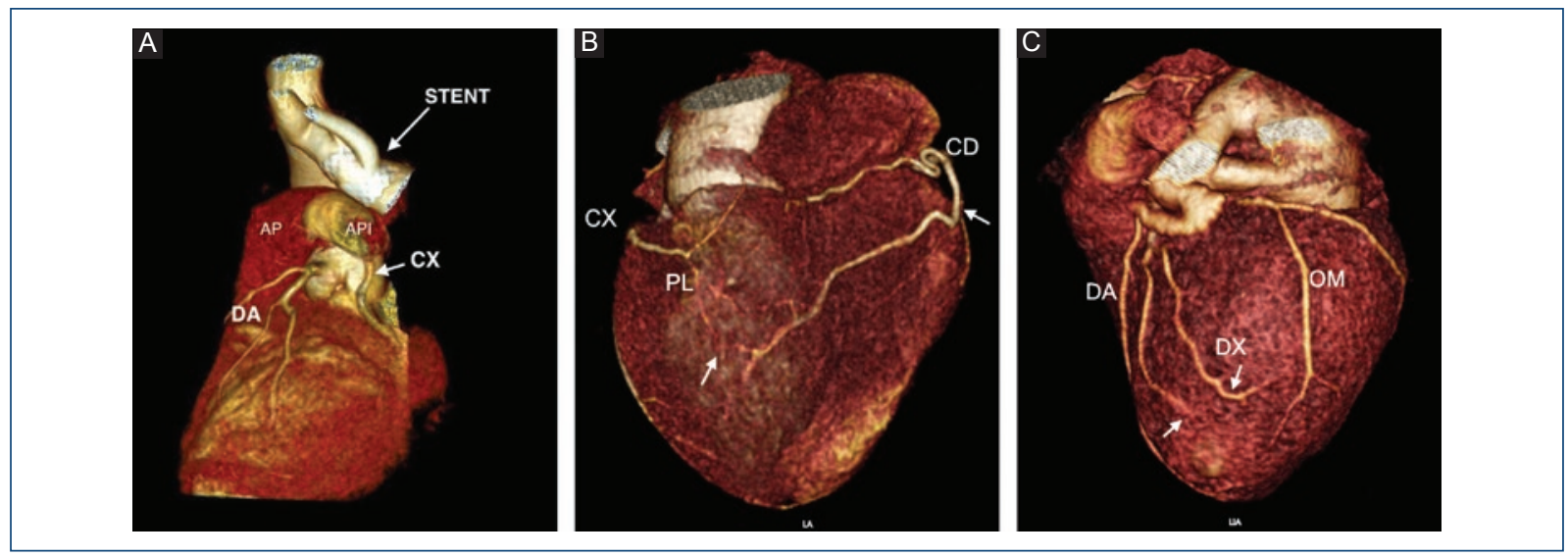

Figura 2. Reconstrucción volumétrica. A: vista lateral izquierda. Se observa el stent debajo de la arteria subclavia izquierda. Nótese origen de CX y relación con la DA con origen y trayecto conservado y su primera diagonal de buen calibre y desarrollo. B: vista cara inferior, muestra ramo agudo marginal de buen desarrollo y calibre, brindando circulación hacia territorio de PL. C: vista lateral izquierda, se observan primer y segundo ramos diagonales de buen desarrollo brindando la circulación colateral hacia el territorio del ramo $0 \mathrm{M}$.

$\mathrm{AP}$ : tronco de la arteria pulmonar; API: arteria pulmonar izquierda; CD: coronaria derecha; CX: coronaria circunfleja; DA: descendente anterior; DX: ramos diagonales 1 y 2; PL: ramo posterolateral de la CX; OM: oblicua marginal.

\section{Conflicto de intereses}

Los autores declaran no tener conflicto de intereses.

\section{Responsabilidades éticas}

Protección de personas y animales. Los autores declaran que para esta investigación no se han realizado experimentos en seres humanos ni en animales.

Confidencialidad de los datos. Los autores declaran que han seguido los protocolos de su centro de trabajo sobre la publicación de datos de pacientes.
Derecho a la privacidad y consentimiento informado. Los autores declaran que en este artículo no aparecen datos de pacientes.

\section{Bibliografía}

1. Çitaku H, Kamberi L, Gorani D, Koçinaj D, Krasniqi X. Anomalous origin of left circumflex artery. Med Arch. 2015;69:423-4.

2. Sekelyk R, Mykychak Y, Fedevych O, Yemets I. Anomalous origin of circumflex coronary artery from right pulmonary artery associated with coarctation of the aorta: a case report of surgical treatment. World $\mathrm{J}$ Pediatr Congenit Heart Surg. 2011;5:97-9.

3. Aktas D, Erdem A, Celik N, Kamalı H, Sarıtas T. A rare coronary anomaly with masked diagnosis: Anomalous left circumflex artery from right pulmonary artery. Turk Kardiyol Dern Ars. 2015;43(6):551-3. 